JOURNAL OF THE

AMERICAN MATHEMATICAL SOCIETY

Volume 23, Number 2, April 2010, Pages 591-609

S 0894-0347(09)00652-3

Article electronically published on November 24, 2009

\title{
ON THE ILL-POSEDNESS OF THE PRANDTL EQUATION
}

\author{
DAVID GÉRARD-VARET AND EMMANUEL DORMY
}

\section{INTRODUCTION}

One challenging open problem of fluid dynamics is to understand the inviscid limit of the Navier-Stokes equations

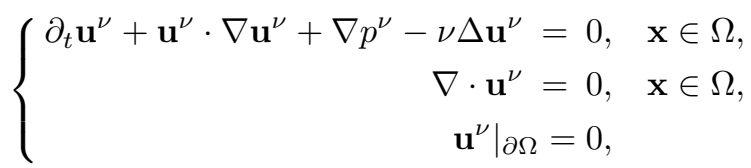

in a domain $\Omega$ with boundaries, endowed with a no-slip boundary condition. Mathematically, the main difficulty is the lack of uniform bounds on the vorticity field, as the viscosity $\nu$ goes to zero. In terms of fluid dynamics, this corresponds to a boundary layer phenomenon near $\partial \Omega$.

A natural approach to describe this boundary layer is to look for a double-scale asymptotics, with a parabolic scaling in the normal direction. Consider the case $\Omega \subset \mathbb{R}^{2}$. At least locally, any point $\mathbf{x}$ in a neighborhood of $\partial \Omega$ has a unique decomposition

$$
\mathbf{x}=y \mathbf{n}(x)+\tilde{\mathbf{x}}(x), \quad \tilde{\mathbf{x}} \in \partial \Omega,
$$

where $y>0, x$ is an arc length parametrization of the boundary, and $\mathbf{n}$ is the inward unit normal vector at $\partial \Omega$. The velocity field can be written

$$
\mathbf{u}^{\nu}(t, \mathbf{x})=u^{\nu}(t, x, y) \boldsymbol{\tau}(x)+v^{\nu}(t, x, y) \mathbf{n}(x),
$$

where $(\boldsymbol{\tau}, \mathbf{n})$ is the Frénet frame. It is then natural to consider an approximation of the type:

$$
\begin{aligned}
& u^{\nu}(t, x, y) \approx u^{0}(t, x, y)+u^{B L}(t, x, y / \sqrt{\nu}), \\
& v^{\nu}(t, x, y) \approx v^{0}(t, x, y)+\sqrt{\nu} v^{B L}(t, x, y / \sqrt{\nu}),
\end{aligned}
$$

where

$$
\mathbf{u}^{0}(t, x, y)=u^{0}(t, x, y) \boldsymbol{\tau}(x)+v^{0}(t, x, y) \mathbf{n}(x)
$$

satisfies the Euler equation with the no penetration condition, and

$$
\left(u^{B L}, v^{B L}\right)=\left(u^{B L}, v^{B L}\right)(t, x, Y)
$$

describes a boundary layer corrector with typical scale $\sqrt{\nu}$ in the normal direction. It is slightly more convenient to introduce

$u(t, x, Y):=u^{0}(t, x, 0)+u^{B L}(t, x, Y), \quad v(t, x, Y):=Y \partial_{y} v^{0}(t, x, 0)+v^{B L}(t, x, Y)$.

Received by the editors April 2, 2009.

2010 Mathematics Subject Classification. Primary 35-XX; Secondary 76-XX.

(C)2009 American Mathematical Society Reverts to public domain 28 years from publication 
Indeed, inserting this Ansatz in the Navier-Stokes equations, we get formally

$$
\left\{\begin{array}{rr}
\partial_{t} u+u \partial_{x} u+v \partial_{Y} u-\partial_{Y}^{2} u=\left.\left(\partial_{t} u^{0}+u^{0} \partial_{x} u^{0}\right)\right|_{y=0}, & Y>0, \\
\partial_{x} u+\partial_{Y} v=0, & Y>0, \\
(u, v)=0, & Y=0, \\
\lim _{Y \rightarrow+\infty} u=\left.u^{0}\right|_{y=0} . &
\end{array}\right.
$$

These are the so-called Prandtl equations, derived by Ludwig Prandtl [12. Note that the curvature of the boundary does not appear explicitly in the system. It is however involved in (1.3) through the Euler field, and through the interval of definition of the arc length parametrization $x$. Up to our knowledge, all studies deal with one of the following three cases: $x \in \mathbb{R}, x \in \mathbb{T}$, or $0<x<L$, supplemented with a condition on $u$ at $x=0$. The first and second choices are convenient to describe phenomena that are local in $x$. The case $x \in \mathbb{T}$ may also model the outside of a bounded convex obstacle. Finally, the third configuration is adapted to the spreading of a flow around a thin obstacle, where $x=0$ corresponds to the tip of the obstacle.

Although this formal asymptotics is very natural, its validity is not clear. As emphasized by physicists, including Prantdl himself, it may not hold uniformly in space and time. One reason is the so-called boundary layer separation, which is observed for flows around obstacles; see [7. Nevertheless, the description (1.2) fits with many experiments, upstream from the separation zone. In any case, to understand the relevance and limitations of the Prandtl model is a crucial issue.

From the mathematical point of view, one must address two problems:

(1) the well-posedness of the Prandtl equation;

(2) the justification of the expansion (1.2).

These two problems depend crucially on the choice of the underlying functional spaces, especially on the regularity that is required in the tangential variable $x$. Indeed, the main mathematical difficulty is the lack of control of the $x$ derivatives. For example, $v$ is recovered in (1.3) through the divergence condition, and in terms of $x$-regularity, behaves broadly like $\partial_{x} u$. This loss of one derivative is not balanced by any horizontal diffusion term, so that standard energy estimates do not apply.

Within spaces of functions that are analytic in $x \in \mathbb{R}, Y \in \mathbb{R}^{+}$, Sammartino and Caflisch have overcome these problems, justifying locally in time the boundary layer asymptotics 13, 14. But for more "realistic" functional settings, the way solutions of (1.1) behave is still poorly understood. Various instability mechanisms, which are filtered out in an analytic framework, become a huge source of trouble. For example, when the viscosity is small, the Navier-Stokes equation admits exponentially growing solutions which are both small-scale and isotropic in $x, y$. Their evolution is lost in the anisotropic Prandtl description.

This remark was used by Emmanuel Grenier in 6, who relied on the so-called Rayleigh instability for inviscid flows to show that the asymptotics (1.2) does not (always) hold in the Sobolev space $H^{1}$ (see [6] for a precise statement). However, the relevance of this asymptotics in $L^{p}$, or its relevance in the absence of Rayleigh instabilities, is still an open issue.

Above all, the local in time well-posedness of the Prandtl equation for smooth (say Sobolev) initial data has been so far an open problem. Up to our knowledge, the Cauchy problem has only been solved in two settings: 
i) $x \in \mathbb{R}$, with data that are analytic in $x:$ see [13, 10] for more;

ii) $0<x<L$, with data that are monotonic in $y$ : see [11, 15] for more.

One may also cite article 4, in which the blowup in time of some smooth solutions is exhibited. Finally, let us mention the interesting work [8], in which the inviscid version of (1.3) is analyzed (no $\partial_{Y}^{2} u$ in the equation). Interestingly, for a smooth initial data, this equation turns out to have an explicit solution through the method of characteristics. In particular, starting from a smooth data, one recovers locally in time a smooth data. More precisely, there is only a finite loss of $x$-derivatives, so that the Cauchy problem is (weakly) well-posed. We refer to $[8$ for all details. See also papers [5, 1] on the hydrostatic equations, which share some features with Prandtl equations. For more on Prandtl equations, see the review [3].

On the basis of the inviscid result, it seems reasonable to bet for well-posedness of the Prandtl equation (1.3) in Sobolev type spaces. The aim of this paper is to show that it is actually linearly ill-posed in this framework. As we shall see later on, the reason for ill-posedness is a strong destabilization mechanism due to two ingredients: viscosity, and critical points in the base velocity profile. In particular, it does not contradict the positive results obtained in the inviscid case and for monotonic data.

We now describe our results. We consider the domain $(x, Y) \in \mathbb{T} \times \mathbb{R}^{+}$and restrict to Euler fields with constant slip at the boundary: $\mathbf{u}^{0}(t, x, 0)=(\underline{u}, 0)$, for some real constant $\underline{u}$. To lighten notation, we write $y$ instead of $Y$. The Prandtl equation comes down to

$$
\left\{\begin{array}{rc}
\partial_{t} u+u \partial_{x} u+v \partial_{y} u-\partial_{y}^{2} u=0, & \text { in } \mathbb{T} \times \mathbb{R}^{+}, \\
\partial_{x} u+\partial_{y} v=0, & \text { in } \mathbb{T} \times \mathbb{R}^{+}, \\
\left.(u, v)\right|_{y=0}=(0,0), & \lim _{y \rightarrow+\infty} u=\underline{u} .
\end{array}\right.
$$

Let $u_{s}=u_{s}(t, y)$ be a smooth solution of the heat equation

$$
\partial_{t} u_{s}-\partial_{y}^{2} u_{s}=0,\left.\quad u_{s}\right|_{y=0}=0, \quad \lim _{y \rightarrow+\infty} u_{s}=\underline{u},\left.\quad u_{s}\right|_{t=0}=U_{s} .
$$

Clearly, the shear velocity profile $\left(u_{s}, v_{s}\right)=\left(u_{s}(t, y), 0\right)$ satisfies the system (1.4). We consider the linearization around $\left(u_{s}, v_{s}\right)$, that is,

$$
\left\{\begin{array}{rc}
\partial_{t} u+u_{s} \partial_{x} u+v \partial_{y} u_{s}-\partial_{y}^{2} u=0, & \text { in } \mathbb{T} \times \mathbb{R}^{+}, \\
\partial_{x} u+\partial_{y} v=0, & \text { in } \mathbb{T} \times \mathbb{R}^{+}, \\
\left.(u, v)\right|_{y=0}=(0,0), & \lim _{y \rightarrow+\infty} u=0 .
\end{array}\right.
$$

We wish to study well-posedness properties of (1.6), for a certain class of velocities $u_{s}$. In this view, we introduce the following functional spaces:

$$
W_{\alpha}^{s, \infty}\left(\mathbb{R}^{+}\right):=\left\{f=f(y), \quad e^{\alpha y} f \in W^{s, \infty}\left(\mathbb{R}^{+}\right)\right\}, \quad \forall \alpha, s \geq 0,
$$

with $\|f\|_{W_{\alpha}^{s, \infty}}:=\left\|e^{\alpha y} f\right\|_{W^{s, \infty}}$, and

$$
E_{\alpha, \beta}:=\left\{u=u(x, y)=\sum_{k \in \mathbb{Z}} \hat{u}^{k}(y) e^{i k x},\left\|\hat{u}^{k}\right\|_{W_{\alpha}^{0, \infty}} \leq C_{\alpha, \beta} e^{-\beta|k|}, \forall k\right\}, \forall \alpha, \beta>0,
$$

with $\|u\|_{E_{\alpha, \beta}}:=\sup _{k} e^{\beta|k|}\left\|\hat{u}^{k}\right\|_{W_{\alpha}^{0, \infty}}$. 
Note that the functions of $E_{\alpha, \beta}$ have analytic regularity in $x$. They have only $L^{\infty}$-regularity in $y$, with an exponential weight. More regularity in $y$ could be considered as well. Let $\alpha, \beta>0$. We prove in the appendix the following result:

Proposition 1 (Well-posedness in the analytic setting). Let $u_{s}-\underline{u} \in C^{0}\left(\mathbb{R}_{+}\right.$; $\left.W_{\alpha}^{1, \infty}\left(\mathbb{R}_{+}\right)\right)$. There exists $\rho>0$ such that: for all $T$ with $\beta-\rho T>0$, and all $u_{0} \in E_{\alpha, \beta}$, the linear equation (1.6) has a unique solution

$$
u \in C\left([0, T) ; E_{\alpha, \beta-\rho T}\right), \quad u(t, \cdot) \in E_{\alpha, \beta-\rho t},\left.\quad u\right|_{t=0}=u_{0} .
$$

In short, the Cauchy problem for (1.6) is locally well-posed in the analytic setting. We shall denote

$$
T(t, s) u_{0}:=u(t, \cdot),
$$

where $u$ is the solution of (1.6) with $\left.u\right|_{t=s}=u_{0}$. As the spaces $E_{\alpha, \beta}$ are dense in the spaces

$$
H^{m}:=H^{m}\left(\mathbb{T}_{x}, W_{\alpha}^{0, \infty}\left(\mathbb{R}_{y}^{+}\right)\right), \quad m \geq 0,
$$

this makes sense to introduce the following notation: for all $T \in \mathcal{L}\left(E_{\alpha, \beta}, E_{\alpha, \beta^{\prime}}\right)$,

$$
\|T\|_{\mathcal{L}\left(H^{\left.m_{1}, H^{m_{2}}\right)}\right.}=\sup _{u_{0} \in E_{\alpha, \beta}} \frac{\left\|T u_{0}\right\|_{H^{m_{2}}}}{\left\|u_{0}\right\|_{H^{m_{1}}}}
$$

which belongs to $\mathbb{R}_{+} \cup\{+\infty\}$. In particular, it is infinite when $T$ does not extend to a bounded operator from $H^{m_{1}}$ to $H^{m_{2}}$. The main result of our paper is

Theorem 1 (Ill-posedness in the Sobolev setting).

i) Let $u_{s}-\underline{u} \in C^{0}\left(\mathbb{R}_{+} ; W_{\alpha}^{4, \infty}\left(\mathbb{R}_{+}\right)\right) \cap C^{1}\left(\mathbb{R}_{+} ; W_{\alpha}^{2, \infty}\left(\mathbb{R}_{+}\right)\right)$. Assume that the initial velocity has a nondegenerate critical point over $\mathbb{R}^{+}$. Then, there exists $\sigma>0$, such that for all $\delta>0$,

$$
\sup _{0 \leq s \leq t \leq \delta}\left\|e^{-\sigma(t-s) \sqrt{\left|\partial_{x}\right|}} T(t, s)\right\|_{\mathcal{L}\left(H^{m}, H^{m-\mu}\right)}=+\infty, \forall m \geq 0, \mu \in[0,1 / 2) .
$$

ii) Moreover, one can find solutions $u_{s}$ of (1.5) and $\sigma>0$ such that: for all $\delta>0$,

$$
\sup _{0 \leq s \leq t \leq \delta}\left\|e^{-\sigma(t-s) \sqrt{\left|\partial_{x}\right|}} T(t, s)\right\|_{\mathcal{L}\left(H^{m_{1}}, H^{m_{2}}\right)}=+\infty, \quad \forall m_{1}, m_{2} \geq 0 .
$$

This theorem expresses strong linear ill-posedness of the Prandtl equation in the Sobolev framework. It is a consequence of an instability process, which holds at high tangential frequencies. We will show that some perturbations with tangential frequency $k \gg 1$ grow in the linear régime like $e^{\sqrt{k} t}$. The extension of our instability result to the nonlinear setting is still an open issue. In particular, we are not able to apply Grenier's method to go from linear to nonlinear instability: indeed, it requires upper bounds on $T(t, s)$ that we do not have. However, we believe this difficulty to be purely technical: in our view, it is very unlikely that this destabilization phenomenon can be cancelled by nonlinear interactions.

The outline of the paper is as follows. Section 2 gives a formal description of the instability mechanism. It relies on an asymptotic analysis of (1.6), in the high tangential frequency limit. Thanks to this analysis, we show that ill-posedness for the PDE (1.6) comes down to a "spectral condition" for a reduced ODE, namely: 
(SC) There exists $\tau \in \mathbb{C}$ with $\mathcal{I} m \tau<0$, and a solution $W=W(z)$ of

$$
\left(\tau-z^{2}\right)^{2} \frac{d}{d z} W+i \frac{d^{3}}{d z^{3}}\left(\left(\tau-z^{2}\right) W\right)=0,
$$

such that $\lim _{z \rightarrow-\infty} W=0, \lim _{z \rightarrow+\infty} W=1$.

This spectral condition is studied in section 3 and shown to be satisfied. On these grounds, we prove Theorem 1, $c f$. section 4. We end the paper with numerical computations, which emphasize that our instability mechanism is effective.

\section{The InstabiLITy MECHANiSM}

In this section, we describe the destabilization of system (1.6), leading to the ill-posedness theorem. As we shall see, it takes place at high tangential frequencies, say $O(1 / \varepsilon)$, and has a typical time $O(\sqrt{\varepsilon})$. At this timescale, the time dependence of the base velocity $\left(u_{s}(t, y), 0\right)$ will not play an important role. Thus, to understand the instability mechanism, we can consider the simpler equation

$$
\left\{\begin{aligned}
\partial_{t} u+U_{s} \partial_{x} u+v U_{s}^{\prime}-\partial_{y}^{2} u=0, & \text { in } \mathbb{T} \times \mathbb{R}^{+}, \\
\partial_{x} u+\partial_{y} v=0, & \text { in } \mathbb{T} \times \mathbb{R}^{+}, \\
\left.(u, v)\right|_{y=0}=(0,0) . &
\end{aligned}\right.
$$

Handling of the real equation, that is with $u_{s}$ instead of $U_{s}$, will require minor modifications, to be made in section 4

System (1.6) has constant coefficients in $t$ and $x$, so that we can perform a Fourier analysis: we look for solutions in the form

$$
u(t, y)=e^{i k(\omega(k) t+x)} \hat{u}^{k}(y), \quad v=k e^{i k(\omega(k) t+x)} \hat{v}^{k}(y), \quad k>0 .
$$

As we are interested in high tangential frequencies, we denote $\varepsilon:=1 / k \ll 1$, and write $\omega(\varepsilon)$ instead of $\omega(k), u_{\varepsilon}(y), v_{\varepsilon}(y)$ instead of $\hat{u}^{k}(y), \hat{v}^{k}(y)$. The divergence condition yields $v_{\varepsilon}^{\prime}(y)=-i u_{\varepsilon}(y)$. Using this relation in the first equation in (2.1), one ends up with

$$
\left\{\begin{array}{c}
\left(\omega(\varepsilon)+U_{s}\right) v_{\varepsilon}^{\prime}-U_{s}^{\prime} v_{\varepsilon}+i \varepsilon v_{\varepsilon}^{(3)}=0, \quad y>0, \\
\left.v_{\varepsilon}\right|_{y=0}=\left.v_{\varepsilon}^{\prime}\right|_{y=0}=0 .
\end{array}\right.
$$

Thus, the high frequency limit $\varepsilon \rightarrow 0$ in the variable $x$ yields a singular perturbation problem in the variable $y$. To investigate this problem, one must first consider the inviscid case $\varepsilon=0$.

2.1. The inviscid case. When $\varepsilon=0$, one can a priori only retain the impermeability condition. The appropriate problem is

$$
\left(\omega+U_{s}\right) v^{\prime}-U_{s}^{\prime} v=0, \quad y>0,\left.\quad v\right|_{y=0}=0 .
$$

This spectral problem, as well as the corresponding evolution equation, has been studied exhaustively in [8]. Clearly, there are nontrivial solutions if and only if $\omega$ belongs to the range of $-U_{s}$. Moreover, the couples

$$
\omega_{a}=-U_{s}(a), \quad v_{a}=H(y-a)\left(U_{s}-U_{s}(a)\right), \quad a>0,
$$

where $H$ is the Heaviside function, satisfy (2.4). Note that the regularity of $v_{a}$ depends on the choice of $a$. When $a$ is a critical point, it belongs to $W_{\alpha}^{2, \infty}\left(\mathbb{R}^{+}\right)$ with a discontinuous second derivative. Otherwise, it is only in $W_{\alpha}^{1, \infty}\left(\mathbb{R}^{+}\right)$, with a 
discontinuous first derivative. Luckily enough, the additional boundary condition $\left.v_{a}^{\prime}\right|_{y=0}=0$ is also satisfied.

2.2. The viscous perturbation. When $\varepsilon$ is not 0 , the inviscid eigenelements $\omega_{a}, v_{a}$ do not solve (2.3). All boundary conditions are satisfied, $c f$. the above remark, but the equation is not. First, there is an $O(\varepsilon)$ remaining term for $y>a$. More importantly, $v_{a}$ is not smooth at $y=a$, whereas a solution of this parabolic equation should be.

Nevertheless, at least if $a$ is a nondegenerate critical point, there is an approximate solution near $\left(\omega_{a}, v_{a}\right)$. We shall establish this rigorously in section 4 . We just give here a formal expansion. It reads

$$
\left\{\begin{aligned}
\omega(\varepsilon) & \sim \omega_{a}+\varepsilon^{1 / 2} \tau \\
v_{\varepsilon}(y) & \sim v_{a}+\varepsilon^{1 / 2} \tau H(y-a)+\varepsilon^{1 / 2} V\left(\frac{y-a}{\varepsilon^{1 / 4}}\right)
\end{aligned}\right.
$$

where $\tau \in \mathbb{C}$, and $V=V(z)$ quickly tends to zero as $z \rightarrow \pm \infty$. Note that the approximation of $v_{\varepsilon}$ has two parts: the "regular" part

$$
v_{\varepsilon}^{r e g}(y)=H(y-a)\left(U_{s}(y)-U_{s}(a)+\varepsilon^{1 / 2} \tau\right)
$$

and the "shear layer part"

$$
v_{\varepsilon}^{s l}(y)=\varepsilon^{1 / 2} V\left(\frac{y-a}{\varepsilon^{1 / 4}}\right) .
$$

For $\omega(\varepsilon)=-U_{s}(a)+\varepsilon^{1 / 2} \tau$, the function $v_{\varepsilon}^{r e g}$ solves (2.3) up to $O(\varepsilon)$, away from the critical point $y=a$. However, it has a jump at $y=a$, together with its second derivative. The role of the shear layer $v_{\varepsilon}^{s l}$, which concentrates near $y=a$, is to cancel these discontinuities. Still formally, we obtain the system satisfied by the profile $V$ :

$$
\left\{\begin{array}{l}
\left(\tau+U_{s}^{\prime \prime}(a) \frac{z^{2}}{2}\right) V^{\prime}-U_{s}^{\prime \prime}(a) z V+i V^{(3)}=0, \quad z \neq 0 \\
{[V]_{\left.\right|_{z=0}}=-\tau, \quad\left[V^{\prime}\right]_{\left.\right|_{z=0}}=0, \quad\left[V^{\prime \prime}\right]_{\left.\right|_{z=0}}=-U^{\prime \prime}(a)} \\
\lim _{ \pm \infty} V=0
\end{array}\right.
$$

Let us point out that this system is a priori overdetermined, as jump and boundary conditions provide too many constraints. This justifies the introduction of the parameter $\tau$ in the Ansatz (2.5). As we shall see below, there is a $\tau$ for which system (2.6) has a solution. Moreover, $\mathcal{I} m \tau$ is negative. Hence, back to the Fourier representation (2.2), the $k$-th mode will grow in time like $e^{-\mathcal{I} m \tau \sqrt{k} t}$. This is the key of the instability mechanism.

To see how the condition (SC) of the introduction steps in, we need a few rewritings. First, $\tau+U_{s}^{\prime \prime}(a) \frac{z^{2}}{2}$ satisfies the equation in (2.6). We therefore introduce

$$
\tilde{V}(z)=V(z)+\mathbf{1}_{\mathbb{R}_{+}}\left(\tau+U_{s}^{\prime \prime}(a) \frac{z^{2}}{2}\right),
$$


which leads to

$$
\left\{\begin{array}{l}
\left(\tau+U_{s}^{\prime \prime}(a) \frac{z^{2}}{2}\right) \tilde{V}^{\prime}-U_{s}^{\prime \prime}(a) z \tilde{V}+i \tilde{V}^{(3)}=0, \quad z \in \mathbb{R}, \\
\lim _{-\infty} \tilde{V}=0, \quad \tilde{V} \sim_{+\infty} \tau+U_{s}^{\prime \prime}(a) \frac{z^{2}}{2} .
\end{array}\right.
$$

Then, we introduce $W$ such that

$$
\tilde{V}=\left(\tau+U_{s}^{\prime \prime}(a) z^{2} / 2\right) W .
$$

We get:

$$
\left\{\begin{array}{l}
\left(\tau+U_{s}^{\prime \prime}(a) z^{2} / 2\right)^{2} \frac{d}{d z} W+i \frac{d^{3}}{d z^{3}}\left(\left(\tau+U_{s}^{\prime \prime}(a) z^{2} / 2\right) W\right)=0, \\
\lim _{-\infty} W=0, \quad \lim _{+\infty} W=1 .
\end{array}\right.
$$

Finally, we perform the change of variables

$$
\tau=\frac{1}{\sqrt{2}}\left|U_{s}^{\prime \prime}(a)\right|^{1 / 2} \tilde{\tau}, \quad z=2^{1 / 4}\left|U_{s}^{\prime \prime}(a)\right|^{-1 / 4} \tilde{z} .
$$

Dropping the tildes leaves us with the reduced ODE

$$
\left\{\begin{array}{l}
\left(\tau+\operatorname{sign}\left(U_{s}^{\prime \prime}(a)\right) z^{2}\right)^{2} \frac{d}{d z} W+i \frac{d^{3}}{d z^{3}}\left(\left(\tau+\operatorname{sign}\left(U_{s}^{\prime \prime}(a)\right) z^{2}\right) W\right)=0, \\
\lim _{-\infty} W=0, \quad \lim _{+\infty} W=1 .
\end{array}\right.
$$

If $U_{s}^{\prime \prime}(a)<0$, it is exactly the system in (SC). If on the contrary $U_{s}^{\prime \prime}(a)>0$, and if $(\tau, W)$ satisfies the system in (SC), then $(\tau:=-\bar{\tau}, W:=\bar{W})$ satisfies the above system. In both cases, back to the original system (2.6), condition (SC) gives a solution $(\tau, V)$ with $\mathcal{I} m \tau<0$. In particular, this $\sqrt{\varepsilon}$ correction to the eigenvalue is a source of strong instability, leading to ill-posedness.

The proof of Theorem 1, which is based on this formal shear layer phenomenon, is postponed to section 4 In the next paragraph, we focus on condition (SC) and prove that it is satisfied.

\section{The spectral CONDition (SC)}

We need to study the existence of heteroclinic orbits for the ODE (1.7). Note that $W=1$ is a solution. Equation (1.7) can be written as a second order equation in $X=W^{\prime}$ :

$$
i\left(\tau-z^{2}\right) X^{\prime \prime}-6 i z X^{\prime}+\left(\left(\tau-z^{2}\right)^{2}-6 i\right) X=0 .
$$

To show that (SC) holds, we proceed in three steps.

Step 1. We consider an auxiliary eigenvalue problem:

$$
A u:=\frac{1}{z^{2}+1} u^{\prime \prime}+\frac{6 z}{\left(z^{2}+1\right)^{2}} u^{\prime}+\frac{6}{\left(z^{2}+1\right)^{2}} u=\alpha u .
$$

For its study, we introduce the weighted spaces

$$
\begin{aligned}
\mathcal{L}^{2} & :=\left\{u \in L_{\text {loc }}^{2}, \int_{\mathbb{R}}\left(z^{2}+1\right)^{4}|u|^{2}<+\infty\right\}, \\
\mathcal{H}^{1} & :=\left\{u \in H_{\text {loc }}^{1} \int_{\mathbb{R}}\left(z^{2}+1\right)^{4}|u|^{2}+\int_{\mathbb{R}}\left(z^{2}+1\right)^{3}\left|u^{\prime}\right|^{2}<+\infty\right\} \hookrightarrow \mathcal{L}^{2},
\end{aligned}
$$


with their obvious Hilbert norms. We see $A$ as an operator from $D(A):=\left\{u \in \mathcal{H}^{1}\right.$, $\left.A u \in \mathcal{L}^{2}\right\}$ into $\mathcal{L}^{2}$. Our goal is to show that $A$ has a positive eigenvalue.

By standard arguments, the domain $D(A)$ is dense in $\mathcal{L}^{2}$. Moreover, for any $u$ in $D(A)$, there is a sequence $u_{n}$ of smooth functions with compact support, such that $u_{n} \rightarrow u$ in $\mathcal{H}^{1}$ and $A u_{n} \rightarrow A u$ in $\mathcal{L}^{2}$. Integration by parts and use of this density property give easily that $A$ is symmetric, i.e.

$$
\forall u, v \in D(A), \quad(A u \mid v)_{\mathcal{L}^{2}}=(A v \mid u)_{\mathcal{L}^{2}},
$$

and that for $\lambda$ large enough,

$((\lambda-A) u \mid u)_{\mathcal{L}^{2}}=\lambda \int_{\mathbb{R}}\left(z^{2}+1\right)^{4}|u|^{2}-6 \int_{\mathbb{R}}\left(z^{2}+1\right)^{2}|u|^{2}+\int_{\mathbb{R}}\left(z^{2}+1\right)^{3}\left|u^{\prime}\right|^{2} \geq \frac{1}{2}\|u\|_{\mathcal{H}^{1}}^{2}$.

Then, the coercivity condition (3.4) allows us to apply the Lax-Milgram lemma. It implies the invertibility of $\lambda-A$, with

$$
\left\|(\lambda-A)^{-1} f\right\|_{\mathcal{L}^{2}} \leq\left\|(\lambda-A)^{-1} f\right\|_{\mathcal{H}^{1}} \leq C\|f\|_{\mathcal{L}^{2}} .
$$

Moreover, from (3.3), $(\lambda-A)^{-1}$ is selfadjoint, and so is $A$.

We now prove that $A$ has positive spectrum. To do so, we claim that it is enough to find $u \in D(A)$ with $(A u \mid u)_{\mathcal{L}^{2}}>0$. Indeed, suppose a contrario that $\sigma(A)$ is contained in $\mathbb{R}_{-}$. Then, by the spectral theorem,

$$
\forall \alpha>0, \quad\left\|(A-\alpha)^{-1}\right\|=\frac{1}{d(\alpha, \sigma(A))} \leq \alpha^{-1} .
$$

We deduce: for all $u \in D(A)$,

$$
\|u\|_{\mathcal{L}^{2}}^{2} \leq \alpha^{-2}\|(A-\alpha) u\|_{\mathcal{L}^{2}}^{2} .
$$

Expanding the scalar products, we obtain

$$
0 \leq \alpha^{-2}\|A u\|_{\mathcal{L}^{2}}^{2}-2 \alpha^{-1}(A u \mid u)_{\mathcal{L}^{2}} .
$$

In the limit $\alpha \rightarrow+\infty$, we get $(A u \mid u)_{\mathcal{L}^{2}} \leq 0$ for all $u \in D(A)$. This proves our claim. From there, we simply take $u=e^{-2 z^{2}}$. A straightforward computation gives

$$
(A u \mid u)=\frac{439}{512} \sqrt{\pi}>0,
$$

and so $\sigma(A)$ has a positive subset.

It remains to exhibit a positive eigenvalue inside this positive subset of the spectrum. We remark that the operator $A$ can be split into

$$
A=A_{1}+A_{2}, \quad A_{1} u:=\frac{1}{z^{2}+1} u^{\prime \prime}+\frac{6 z}{\left(z^{2}+1\right)^{2}} u^{\prime}, \quad A_{2} u:=\frac{6}{\left(z^{2}+1\right)^{2}} u .
$$

On the one hand, the operator $A_{1}$ is negative, and by the Lax-Milgram Lemma, $A_{1}-\lambda$ is invertible for any $\lambda>0$. Thus, $\sigma\left(A_{1}\right) \subset \mathbb{R}_{-}$. On the other hand, let $u_{n}$ be a sequence with $u_{n}$ and $A_{1} u_{n}$ bounded in $\mathcal{L}^{2}$. This implies that $u_{n}$ is bounded in $\mathcal{H}^{1}$, and so has a convergent subsequence in $L_{\text {loc }}^{2}$. Moreover, $\left|u_{n}\right|^{2}$ is equi-integrable over $\mathbb{R}$. Finally, it implies that $A_{2} u_{n}$ has a convergent subsequence in $\mathcal{L}^{2}$, which means that $A_{2}$ is $A_{1}$-compact. Hence, the essential spectra of $A$ and $A_{1}$ are the same; see [9]. In particular, the positive part of $\sigma(A)$ is made of isolated eigenvalues with finite multiplicity. Eventually, we state: there exists $\alpha>0$, and $u$ in $D(A)$ satisfying (3.2). 
Step 2. We wish to convert the eigenelements $(\alpha, u)$ of the previous step into an appropriate solution $(\tau, X)$ of (3.1). We set $\tilde{\tau}=-\alpha^{1 / 2}$, and $\tilde{z}=\alpha^{-1 / 4} z, Y(\tilde{z})=$ $u(z)$. Dropping the tildes, we obtain a solution of

$$
\left(\tau-z^{2}\right) Y^{\prime \prime}-6 z Y^{\prime}+\left(\left(\tau-z^{2}\right)^{2}-6\right) Y=0
$$

By a classical bootstrap argument, $Y$ is smooth. Moreover, it inherits from $u$ its integrability properties at infinity. Actually, the behaviour of $Y$ can be further specified, as shown in:

Proposition 2. The function $Y$ admits a unique extension, still denoted by $Y$, that is holomorphic in $z$ and satisfies (3.5) in the simply connected domain

$$
U_{\tau}:=\mathbb{C} \backslash\left(\left[-i \infty,-i|\tau|^{1 / 2}\right] \cup\left[i|\tau|^{1 / 2},+i \infty\right]\right) .
$$

Moreover, in the sectors $\arg z \in(-\pi / 4+\delta, \pi / 4-\delta)$ and $\arg z \in(3 \pi / 4+\delta, 5 \pi / 4-\delta)$, $\delta>0$, it satisfies the inequality

$$
|Y(z)| \leq C_{\delta} \exp \left(-z^{2} / 4\right)
$$

Proof. This proposition follows from the general theory of ODE's with holomorphic coefficients. The existence of a holomorphic solution is well known, because the coefficient $\tau-z^{2}$ does not vanish on $U_{\tau}$. As regards the inequality, we rewrite equation (3.1) as the first order system:

$$
\frac{d}{d z} \mathcal{Y}=z \mathcal{A}(z) \mathcal{Y}, \quad \mathcal{Y}=\left(\begin{array}{c}
Y \\
z^{-1} \frac{d}{d z} Y
\end{array}\right), \quad \mathcal{A}(z)=\left(\begin{array}{c}
0 \\
\frac{6-\left(\tau-z^{2}\right)^{2}}{z^{2}\left(\tau-z^{2}\right)} \frac{6}{\tau-z^{2}}-\frac{1}{z^{2}}
\end{array}\right) .
$$

In particular, $\mathcal{A}$ is holomorphic at infinity, with $\mathcal{A}(\infty)=\left(\begin{array}{ll}0 & 1 \\ 1 & 0\end{array}\right)$. It has two distinct eigenvalues \pm 1 , with eigenvectors $\left(\begin{array}{c}1 \\ \pm 1\end{array}\right)$.

Hence, we can apply [2, Theorem 5.1, p. 163]: in any closed sector $S$ inside which $\Re z^{2}$ does not cancel, there exist solutions $\mathcal{Y}_{ \pm}$(depending a priori on $S$ ) with the following asymptotic behaviour as $|z| \rightarrow+\infty$ :

$$
\mathcal{Y}_{ \pm} \sim\left(\sum_{i \geq 0} \mathcal{Y}_{ \pm}^{i} z^{\alpha_{ \pm}-i}\right) e^{P_{ \pm}(z)},
$$

where $\alpha_{ \pm}$is a complex constant, and $P_{ \pm}(z)$ is a polynomial of degree 2. Moreover, the leading term of $P_{ \pm}$is $\pm \frac{z^{2}}{2}$. Following the same scheme of proof, we get in the present case:

$$
\mathcal{Y}_{ \pm}^{0}=\left(\begin{array}{c}
1 \\
\pm 1
\end{array}\right), \quad \alpha_{ \pm}=-\frac{1}{2}( \pm \tau+7), \quad P_{ \pm}= \pm z^{2} / 2 .
$$

As our solution $Y$ is integrable over $\mathbb{R}$, it is necessarily proportional to the decaying solution. The bounds in Proposition 2 follow.

Now, as $Y$ is defined on $U_{\tau}$, we can perform the complex change of variables:

$$
\tilde{z}:=e^{i \pi / 8} z, \quad \tilde{\tau}:=e^{i \pi / 4} \tau
$$

Note that $\tilde{\tau}$ has a negative imaginary part. Moreover, for $\tilde{z}$ real, the original variable $z$ belongs to the sectors $\arg z \in(-\pi / 4+\delta, \pi / 4-\delta)$ or $\arg z \in(3 \pi / 4+\delta, 5 \pi / 4-\delta)$, with $\delta=1 / 16$. By Proposition 2, the function $X(\tilde{z})=Y(z)$ satisfies the estimate $|X(\tilde{z})| \leq C \exp \left(-\tilde{z}^{2} / 4\right)$. Finally, dropping the tildes yields a solution $\tau, X$ of (3.1), where $X$ decays at infinity. This concludes Step 2 . 
Step 3. To deduce from the previous step that (SC) holds, it is enough that $\int_{\mathbb{R}} X(z) d z$ be nonzero. If so, one can consider

$$
W(z):=\left(\int_{\mathbb{R}} X\left(z^{\prime}\right) d z^{\prime}\right)^{-1} \int_{-\infty}^{z} X\left(z^{\prime}\right) d z^{\prime},
$$

which clearly satisfies all the requirements.

Let us assume a contrario that $\int_{\mathbb{R}} X(z) d z=0$. Then, the function

$$
V(z):=\left(\tau-z^{2}\right) \int_{-\infty}^{z} X\left(z^{\prime}\right) d z^{\prime}
$$

is a solution of

$$
\left(\tau-z^{2}\right) V^{\prime}+2 z V+i V^{(3)}=0,
$$

which decays exponentially as $z$ goes to $\pm \infty$, together with all its derivatives. Differentiation of the equation gives

$$
\left(\tau-z^{2}\right) V^{\prime \prime}+2 V+i V^{(4)}=0 .
$$

Then, we multiply by $\overline{V^{\prime \prime}}$, which is the complex conjugate of $V^{\prime \prime}$, and integrate over $\mathbb{R}$. Simple integrations by parts yield:

$$
\int_{\mathbb{R}}\left(\tau-z^{2}\right)\left|V^{\prime \prime}\right|^{2}-2 \int_{\mathbb{R}}\left|V^{\prime}\right|^{2}-i \int_{\mathbb{R}}\left|V^{(3)}\right|^{2}=0 .
$$

The imaginary part of this identity yields

$$
\mathcal{I} m \tau \int_{\mathbb{R}}\left|V^{\prime \prime}\right|^{2}=\int_{R}\left|V^{(3)}\right|^{2},
$$

which contradicts the fact that $\mathcal{I} m \tau<0$. Thus, the condition $(S C)$ is satisfied.

\section{Proof of ILL-POSEDNESS}

Theorem 1 will be deduced from the formal analysis of section 2, This analysis was performed on (2.1), in which possible time variations of $u_{s}$ were neglected. To account for the original system (1.6) will require a few modifications, notably in the choice of the approximation (2.5). We will distinguish between the parts i) and ii) of the theorem.

4.1. Ill-posedness for general $u_{s}$. Let $u_{s}$ satisfy the assumptions of part i). Let $a$ be the nondegenerate critical point of $\left.u_{s}\right|_{t=0}=U_{s}$. For the sake of brevity, we consider the case $U_{s}^{\prime \prime}(a)<0$, the other one being strictly similar. The differential equation

$$
\partial_{t} \partial_{y} u_{s}(t, a(t))+\partial_{y}^{2} u_{s}(t, a(t)) a^{\prime}(t)=0, \quad a(0)=a
$$

defines for small time $t<t_{0}$ a nondegenerate critical point $a(t)$ of $u_{s}(t, \cdot)$. Then let $\tau, W$ be given by condition (SC). We set

$$
V:=\left(\tau-z^{2}\right) W-\mathbf{1}_{\mathbb{R}_{+}}\left(\tau-z^{2}\right) .
$$

In light of section 2, we introduce, for $\varepsilon>0$ and $t<t_{0}$ :

$$
\omega(\varepsilon, t):=-u_{s}(t, a(t))+\frac{\varepsilon^{1 / 2}}{\sqrt{2}}\left|\partial_{y}^{2} u_{s}(t, a(t))\right|^{1 / 2} \tau
$$

as well as the "regular" velocity

$$
v_{\varepsilon}^{r e g}(t, y):=H(y-a(t))\left(u_{s}(t, y)-u_{s}(t, a(t))+\frac{\varepsilon^{1 / 2}}{\sqrt{2}}\left|\partial_{y}^{2} u_{s}(t, a(t))\right|^{1 / 2} \tau\right),
$$


and the shear layer velocity

$$
v_{\varepsilon}^{s l}(t, y):=\frac{\varepsilon^{1 / 2}}{\sqrt{2}} \varphi(y-a(t))\left|\partial_{y}^{2} u_{s}(t, a(t))\right|^{1 / 2} V\left(\left|\partial_{y}^{2} u_{s}(t, a(t))\right|^{1 / 4} \frac{(y-a(t))}{(2 \varepsilon)^{1 / 4}}\right),
$$

where $\varphi$ is a smooth truncation function near 0 . We then consider the following velocity field:

$$
\begin{aligned}
& u_{\varepsilon}(t, x, y):=e^{i \varepsilon^{-1} x} U_{\varepsilon}(t, y), \quad U_{\varepsilon}(t, y)=i e^{i \varepsilon^{-1} \int_{0}^{t} \omega(\varepsilon, s) d s} \partial_{y}\left(v_{\varepsilon}^{r e g}(t, y)+v_{\varepsilon}^{s l}(t, y)\right), \\
& v_{\varepsilon}(t, x, y):=e^{i \varepsilon^{-1} x} V_{\varepsilon}(t, y), \quad V_{\varepsilon}(t, y)=\varepsilon^{-1} e^{i \varepsilon^{-1} \int_{0}^{t} \omega(\varepsilon, s) d s}\left(v_{\varepsilon}^{r e g}(t, y)+v_{\varepsilon}^{s l}(t, y)\right) .
\end{aligned}
$$

In order to have a field that is $2 \pi$-periodic in $x$ and growing in time, we take $\varepsilon:=\frac{1}{n}$, with $n \in \mathbb{N}_{*}$. One verifies easily that $u_{\varepsilon}=e^{i \varepsilon^{-1} x} U_{\varepsilon}(t, y)$ is analytic in $x$, and $W^{2, \infty}$ in $t, y$. Moreover, we have the bounds

$$
c e^{\frac{\sigma_{0} t}{\sqrt{\varepsilon}}} \leq\left\|U_{\varepsilon}(t, \cdot)\right\|_{W_{\alpha}^{2, \infty}} \leq C e^{\frac{\sigma_{0} t}{\sqrt{\varepsilon}}}
$$

for positive constants $c, C$ and $\sigma_{0}$ that do not depend on $\varepsilon$.

Inserting the expression for $u_{\varepsilon}, v_{\varepsilon}$ into the linearized Prandtl equation (1.6), we obtain

$$
\left\{\begin{array}{rr}
\partial_{t} u_{\varepsilon}+u_{s} \partial_{x} u_{\varepsilon}+v_{\varepsilon} \partial_{y} u_{s}-\partial_{y}^{2} u_{\varepsilon}=r_{\varepsilon}, & \text { in } \mathbb{T} \times \mathbb{R}^{+}, \\
\partial_{x} u_{\varepsilon}+\partial_{y} v_{\varepsilon}=0, & \text { in } \mathbb{T} \times \mathbb{R}^{+}, \\
\left.(u, v)\right|_{y=0}=(0,0) . &
\end{array}\right.
$$

The remainder term $r_{\varepsilon}$ reads $r_{\varepsilon}=e^{i \varepsilon^{-1} x} R_{\varepsilon}(t, y)$, with

$$
\begin{aligned}
R_{\varepsilon}(t, y)=e^{i \varepsilon^{-1} \int_{0}^{t} \omega(\varepsilon, s) d s}( & -\varepsilon^{-1}\left(u_{s}(t, y)-u_{s}(t, a(t))-\partial_{y}^{2} u_{s}(t, a(t)) \frac{y^{2}}{2}\right) \partial_{y} v_{\varepsilon}^{s l}(t, y) \\
& +\varepsilon^{-1}\left(\partial_{y} u_{s}(t, a(t))-\partial_{y}^{2} u_{s}(t, a(t)) y\right) v_{\varepsilon}^{s l}(t, y) \\
& -i \varepsilon \partial_{y}^{3} v_{\varepsilon}^{r e g}(t, y)+i \partial_{t} \partial_{y}\left(v_{\varepsilon}^{r e g}(t, y)+v_{\varepsilon}^{s l}(t, y)\right) \\
& \left.+O\left(\varepsilon^{\infty} e^{\frac{\sigma_{0} t}{\sqrt{\varepsilon}}}\right)\right) .
\end{aligned}
$$

The $O\left(\varepsilon^{\infty}\right)$ gathers terms with derivatives of $\varphi$ : as the shear layer profile $V$ decreases exponentially, and the derivatives of $\varphi(\cdot-a)$ are supported away from $a$, their contribution is indeed exponentially small. Straightforwardly,

$$
\left\|R_{\varepsilon}(t, \cdot)\right\|_{W_{\alpha}^{0, \infty}} \leq C e^{\frac{\sigma_{0} t}{\sqrt{\varepsilon}}}
$$

with the same $\sigma_{0}$ as in (4.1).

We are now in a position to prove part i) of Theorem 1 Let us assume a contrario that for all $\sigma>0$, there exists $m \geq 0, \mu \in[0,1 / 2)$ and $\delta>0$ such that

$$
\sup _{0 \leq s \leq t \leq \delta}\left\|e^{-\sigma(t-s) \sqrt{\left|\partial_{x}\right|}} T(t, s)\right\|_{\mathcal{L}\left(H^{m}, H^{m-\mu}\right)}<+\infty .
$$

Let

$$
T_{\varepsilon}(t, s): W_{\alpha}^{0, \infty}\left(\mathbb{R}_{+}\right) \mapsto W_{\alpha}^{0, \infty}\left(\mathbb{R}_{+}\right)
$$

be the restriction of $T(t, s)$ to the tangential Fourier mode $\varepsilon^{-1}$, namely,

$$
T(t, s)\left(e^{i \varepsilon^{-1} x} U_{0}\right)=e^{i \varepsilon^{-1} x} T_{\varepsilon}(t, s) U_{0} .
$$


Similarly, we denote $L_{\varepsilon}=e^{-i \varepsilon^{-1} x} L e^{i \varepsilon^{-1} x}$, where $L$ is the linearized Prandtl operator around $u_{s}$. We have, for all $0 \leq s \leq t \leq \delta$,

$$
\|T(t, s)\|_{\mathcal{L}\left(W_{\alpha}^{0, \infty}\right)} \leq C \varepsilon^{-\mu} e^{\frac{\sigma(t-s)}{\sqrt{\varepsilon}}} .
$$

Let $U=U(t, y)$ be the solution of $\partial_{t} U+L_{\varepsilon} U=0$ that coincides initially with the approximation $U_{\varepsilon}$. On the one hand, we get

$$
\|U(t, \cdot)\|_{W_{\alpha}^{0, \infty}} \leq C \varepsilon^{-\mu} e^{\frac{\sigma t}{\sqrt{\varepsilon}}}\|U(0, \cdot)\|_{W_{\alpha}^{0, \infty}} \leq C^{\prime} \varepsilon^{-\mu} e^{\frac{\sigma t}{\sqrt{\varepsilon}}} .
$$

On the other hand, the difference $\tilde{U}=U-U_{\varepsilon}$ satisfies, for all $t<\delta$,

$$
\tilde{U}(t, \cdot)=\int_{0}^{t} T_{\varepsilon}(t, s) R_{\varepsilon}(s) d s .
$$

Estimate (4.3) implies that

$$
\|\tilde{U}(t, \cdot)\|_{W_{\alpha}^{0, \infty}} \leq C \varepsilon^{-\mu} \int_{0}^{t} e^{\frac{\sigma(t-s)}{\sqrt{\varepsilon}}} e^{\frac{\sigma_{0} s}{\sqrt{\varepsilon}}} d s \leq C^{\prime} \varepsilon^{1 / 2-\mu} e^{\frac{\sigma_{0} t}{\sqrt{|\varepsilon|}}},
$$

as soon as $\sigma<\sigma_{0}$. Combining this with the estimate (4.1), we obtain the lower bound

$$
\begin{aligned}
\|U(t, \cdot)\|_{W_{\alpha}^{0, \infty}} & \geq\left\|U_{\varepsilon}(t, \cdot)\right\|_{W_{\alpha}^{0, \infty}}-\|\tilde{U}(t, \cdot)\|_{W_{\alpha}^{0, \infty}} \\
& \geq c e^{\frac{\sigma_{0} t}{\sqrt{\varepsilon}}}-C \varepsilon^{\mu-1 / 2} e^{\frac{\sigma_{0} t}{\sqrt{\varepsilon}}} .
\end{aligned}
$$

For $\varepsilon$ small enough, we get

$$
\|U(t, \cdot)\|_{W_{\alpha}^{0, \infty}} \geq c^{\prime} e^{\frac{\sigma_{0} t}{\sqrt{\varepsilon}}}
$$

which contradicts the upper bound (4.4), as soon as $\sigma<\sigma_{0}$ and $t \gg \frac{\mu}{\sigma_{0}-\sigma}|\ln (\varepsilon)| \sqrt{\varepsilon}$. This achieves the proof of part i).

4.2. Stronger ill-posedness for specific $u_{s}$. It remains to handle part ii) of Theorem 1. Roughly, we must find some $u_{s}$ for which $e^{-\sigma \sqrt{\left|\partial_{x}\right|}(t-s)} T(t, s)$ fails to be bounded from $H^{m}$ to $H^{m-\mu}, \mu \geq 0$ arbitrary. Using the notation of the previous paragraph, the key point is to build, for any $N$, a growing solution $U_{\varepsilon, N}$ of

$$
\partial_{t} U_{\varepsilon, N}+L_{\varepsilon} U_{\varepsilon, N}=R_{\varepsilon, N}, \quad \text { where }\left\|R_{\varepsilon, N}(t, \cdot)\right\|_{W_{\alpha}^{0, \infty}} \leq C_{N}\left(\varepsilon^{N}+t^{2 N}\right) e^{\frac{\sigma_{0} t}{\sqrt{\varepsilon}}} .
$$

Indeed, we can then take $N+1 / 2>\mu$ and conclude along the same lines as above.

So far, we have not managed to improve the approximation of the previous paragraph for general $u_{s}$. This explains the technical restriction $\mu \in[0,1 / 2)$ of part i). In order to obtain a refined approximation, we consider some special profiles: we assume that $u_{s}(0, y)=U_{s}(y)$, where $U_{s}$ converges exponentially to $\bar{u}$ and satisfies in the neighborhood of $a>0$ :

$$
U_{s}(y)=U_{s}^{\prime \prime}(a) \frac{(y-a)^{2}}{2}, \quad U_{s}^{\prime \prime}(a)<0 .
$$

Notice that $a$ is a nondegenerate critical point of $U_{s}$. For such profiles, the approximation of the previous paragraph reads

$$
U_{\varepsilon}(t, y)=i e^{i \varepsilon^{-1} \int_{0}^{t} \omega(\varepsilon, s) d s} \partial_{y}\left(v_{\varepsilon}^{r e g}(t, y)+v_{\varepsilon}^{s l}(t, y)\right), \quad \varepsilon=\frac{1}{n}, \quad n \in \mathbb{N}_{*} .
$$


Using that $U_{s}$ is quadratic near $y=a$, one can improve this approximation through an expansion of the type

$$
U_{\varepsilon, N}(t, y)=U_{\varepsilon}(t, y)+i e^{i \varepsilon^{-1} \int_{0}^{t} \omega(\varepsilon, s) d s} \partial_{y} \sum_{i=1}^{N} \varepsilon^{i} v_{\varepsilon}^{i, r e g}(t, y),
$$

with additional terms $v_{\varepsilon}^{i, r e g}$. Let us briefly explain the construction of these extra terms. The error terms due to $U_{\varepsilon}$ can be divided into three categories:

(1) Shear layer terms involving derivatives of $\varphi$. As mentioned before, they are $O\left(\varepsilon^{\infty} e^{\frac{\sigma_{0} t}{\sqrt{\varepsilon}}}\right)$ and require no correction.

(2) Terms that come from the replacement of $u_{s}$ by its Taylor expansion in the shear layer equation. They read

$$
\begin{aligned}
& R_{\varepsilon, 1}:=-\varepsilon^{-1} e^{i \varepsilon^{-1} \int_{0}^{t} \omega(\varepsilon, s) d s}\left(u_{s}(t, y)-u_{s}(t, a(t))-\partial_{y}^{2} u_{s}(t, a(t)) \frac{y^{2}}{2}\right) \partial_{y} v_{\varepsilon}^{s l}(t, y), \\
& R_{\varepsilon, 2}:=\varepsilon^{-1} e^{i \varepsilon^{-1} \int_{0}^{t} \omega(\varepsilon, s) d s}\left(\partial_{y} u_{s}(t, a(t))-\partial_{y}^{2} u_{s}(t, a(t)) y\right) v_{\varepsilon}^{s l}(t, y) .
\end{aligned}
$$

We write

$$
\begin{aligned}
& \left|R_{\varepsilon, 1}\right|=\varepsilon^{-1} e^{\frac{\sigma_{0} t}{\sqrt{\varepsilon}}}\left|\int_{a(t)}^{y} \frac{(z-a(t))^{2}}{2} \partial_{y}^{3} u_{s}(t, z) d z\right|\left|\partial_{y} v_{\varepsilon}^{s l}(t, y)\right| \\
& \leq \varepsilon^{-1} e^{\frac{\sigma_{0} t}{\sqrt{\varepsilon}}} \int_{a(t)}^{y} \frac{(z-a(t))^{2}}{2} \sum_{k=0}^{2 N-1} \frac{t^{k}}{k !}\left|\partial_{t}^{k} \partial_{y}^{3} u_{s}(0, z)\right| d z\left|\partial_{y} v_{\varepsilon}^{s l}(t, y)\right|+O\left(t^{2 N}\right) e^{\frac{\sigma_{0} t}{\sqrt{\varepsilon}}} \\
& \leq \varepsilon^{-1} e^{\frac{\sigma_{0} t}{\sqrt{\varepsilon}}} \int_{a(t)}^{y} \frac{(z-a(t))^{2}}{2} \sum_{k=0}^{2 N-1} \frac{t^{k}}{k !}\left|\partial_{y}^{3+2 k} U_{s}(z)\right| d z\left|\partial_{y} v_{\varepsilon}^{s l}(t, y)\right|+O\left(t^{2 N}\right) e^{\frac{\sigma_{0} t}{\sqrt{\varepsilon}}}
\end{aligned}
$$

The second inequality stems from a Taylor expansion of $u_{s}$ with respect to $t$. As $u_{s}$ satisfies the heat equation, each time derivative can be replaced by two space derivatives, hence the third line. Because $U_{s}$ is quadratic in a vicinity of $a(t)$ (for short times), and $v_{\varepsilon}^{s l}$ and its derivatives decay exponentially fast, we end up with

$$
\left|R_{\varepsilon, 1}\right| \leq C\left(t^{2 N}+\varepsilon^{N}\right) e^{\frac{\sigma_{0} t}{\sqrt{\varepsilon}}}
$$

A similar bound holds for $R_{\varepsilon, 2}$. Hence, these remainders do not require correction.

(3) Terms that come from the time derivative and the diffusion. We focus here on the time derivative, as the diffusion term is simpler and has smaller amplitude. This is

$$
R_{\varepsilon, 3}:=i e^{i \varepsilon^{-1} \int_{0}^{t} \omega(\varepsilon, s) d s} \partial_{t} \partial_{y}\left(v_{\varepsilon}^{r e g}(t, y)+v_{\varepsilon}^{s l}(t, y)\right) .
$$

Proceeding as for $R_{\varepsilon, 2}$, that is, with Taylor expansions in $t$, leads to

$$
\left|e^{i \varepsilon^{-1} \int_{0}^{t} \omega(\varepsilon, s) d s} \partial_{t} \partial_{y} v_{\varepsilon}^{s l}(t, y)\right| \leq C\left(t^{2 N}+\varepsilon^{N}\right) e^{\frac{\sigma_{0} t}{\sqrt{\varepsilon}}} .
$$

As regards the regular part,

$$
\begin{aligned}
e^{i \varepsilon^{-1} \int_{0}^{t} \omega(\varepsilon, s) d s} \partial_{t} \partial_{y} v_{\varepsilon}^{r e g}(t, y) & =e^{i \varepsilon^{-1} \int_{0}^{t} \omega(\varepsilon, s) d s} H(y-a(t)) \partial_{t} \partial_{y} u_{s} \\
& =e^{i \varepsilon^{-1} \int_{0}^{t} \omega(\varepsilon, s) d s} H(y-a(t)) F(t, y)+O\left(t^{2 N} e^{\frac{\sigma_{0} t}{\sqrt{\varepsilon}}}\right),
\end{aligned}
$$


where

$$
F(t, y):=\sum_{k=0}^{2 N-1} \frac{t^{k}}{k !} \partial_{y}^{3+2 k} U_{s}(y)
$$

comes again from a Taylor expansion in $t$. The nice thing about this $O(1)$ term is that it is identically zero in the vicinity of $y=a(t)$ (for short times). As a result, the Heaviside function $H(y-a(t))$ in front of it does not create any discontinuity, and no extra shear layer term is necessary. One takes care of this source term by the introduction of

$v_{\varepsilon}^{1, r e g}=H(y-a(t))\left(u_{s}(t, y)-\omega(\varepsilon, t)\right) \int_{a(t)}^{y} \frac{F(t, z)}{\left(u_{s}(t, z)-\omega(\varepsilon, t)\right)^{2}} d z$,

so that

$$
U_{\varepsilon}^{1}:=-i \varepsilon e^{i \varepsilon^{-1} \int_{0}^{t} \omega(\varepsilon, s) d s} \partial_{y} v_{\varepsilon}^{1, r e g}
$$

solves

$$
\partial_{t} U_{\varepsilon}^{1}+L_{\varepsilon} U_{\varepsilon}^{1}=i e^{i \varepsilon^{-1} \int_{0}^{t} \omega(\varepsilon, s) d s} H(y-a(t)) F(t, y)+O(\varepsilon) .
$$

Proceeding recursively, we obtain an approximation as accurate as we want. This ends the proof of the theorem.

\section{NumericAL STUDY}

In this last section, we present numerical illustrations of the instability process.

5.1. Numerical test of (SC). To check (SC) numerically, it is more convenient to reformulate it with an Evans function. We know from Step 2 section 3 , that there are solutions $\mathcal{Y}_{ \pm}(z)$ of (3.6) satisfying (3.7)-(3.8). Back to the ODE (3.1), this yields independent solutions $X_{+}(\tau, \cdot)$ and $X_{-}(\tau, \cdot)$ respectively growing and decaying as $z$ goes to $+\infty$. Furthermore, the following asymptotics holds:

$$
\begin{aligned}
X_{ \pm}(\tau, z) & \sim z^{ \pm \frac{i \tau}{2 \lambda}-\frac{7}{2}} \exp \left( \pm \frac{1}{2} \lambda z^{2}\right), \\
\partial_{z} X_{ \pm}(\tau, z) & \sim \pm \lambda z^{ \pm \frac{i \tau}{2 \lambda}-\frac{3}{2}} \exp \left( \pm \frac{1}{2} \lambda z^{2}\right),
\end{aligned}
$$

with $\lambda=\frac{1-i}{\sqrt{2}}$. Thus, the functions

$$
W_{-}(\tau, z):=\int_{z}^{+\infty} X_{-}(\tau, s) d s, \quad W_{+}(\tau, z):=\int_{0}^{z} X_{+}(\tau, s) d s, \quad W_{0}(\tau, z)=1,
$$

seen as functions of $z$, form a basis of solutions of (1.7). They are respectively decaying, growing and constant at $+\infty$. As (1.7) is preserved by the change of variable $z \mapsto-z$, the functions $W_{-}(\tau,-z), W_{+}(\tau,-z), W_{0}$ form a basis as well. They are respectively decaying, growing and constant at $-\infty$. The existence of the heteroclinic orbit is the same as the existence of some constants $A$ and $B$ such that

$$
1=A W_{-}(-z, \tau)+B W_{-}(z, \tau)
$$

for all $z$, or equivalently,

$$
\left(\begin{array}{l}
1 \\
0 \\
0
\end{array}\right) \in \operatorname{Vect}\left(\left(\begin{array}{c}
W_{-}(\tau, 0) \\
\partial_{z} W_{-}(\tau, 0) \\
\partial_{z}^{2} W_{-}(\tau, 0)
\end{array}\right),\left(\begin{array}{c}
W_{-}(\tau, 0) \\
-\partial_{z} W_{-}(\tau, 0) \\
\partial_{z}^{2} W_{-}(\tau, 0)
\end{array}\right)\right) .
$$

This last condition is easily seen to be equivalent to

$$
W_{-}(\tau, 0) \neq 0 \quad \text { and } \quad \partial_{z}^{2} W_{-}(\tau, 0)=0 .
$$


Hence, we must find $\tau$ with $\mathcal{I} m \tau<0$ such that

$$
\int_{0}^{+\infty} X_{-}(\tau, s) d s \neq 0 \quad \text { and } \quad \partial_{z} X_{-}(\tau, 0)=0 .
$$

Moreover, we know from Step 3, section 3 that for $\mathcal{I} m \tau<0$, the constraint $\int_{0}^{+\infty} X_{-} \neq 0$ is satisfied. Finally, the condition (SC) comes down to:

$$
\partial_{z} X_{-}(\tau, 0)=0, \quad \text { for some } \tau \text { with } \mathcal{I} m \tau<0 .
$$

To check this, and get a value for $\tau$, one can use a shooting method. For any $\tau$ and any $z_{0} \gg 1$, one can start from the approximation

$$
X_{-}\left(\tau, z_{0}\right) \approx z_{0}^{\frac{-i \tau}{2 \lambda}-\frac{7}{2}} \exp \left(-\frac{1}{2} \lambda z_{0}^{2}\right), \quad \partial_{z} X_{-}\left(\tau, z_{0}\right) \approx-\lambda z_{0}^{\frac{-i \tau}{2 \lambda}-\frac{3}{2}} \exp \left(-\frac{1}{2} \lambda z_{0}^{2}\right),
$$

and integrate (3.1) backwards using a Runge-Kutta scheme. This gives access to the function $\partial_{z} X_{-}(\tau, z)$, for any $\tau$ and any $z \leq z_{0}$. Then, a Newton-Raphson procedure allows us to find a zero in $\{\mathcal{I} m \tau<0\}$ for the function $\tau \mapsto \partial_{z} X \mathcal{-}_{-}(\tau, 0)$. Using such a procedure, we have found

$$
\tau \approx-0.706-0.706 i \text {. }
$$

Note that this value is proportional to $1+i$, as expected from the analysis.

5.2. Simulation of the instability mechanism. To observe the instability mechanism described in section 2, we have performed direct simulations of system (2.1). More precisely, we have considered the velocity

$$
u_{s}(t, y)=U_{s}(y):=2 y \exp \left(-y^{2}\right)
$$

(already studied in $[8]$ in the inviscid case) and solutions of the type

$$
u_{\varepsilon}(t, x, y)=i e^{i \varepsilon^{-1} x} \partial_{y} V_{\varepsilon}\left(\varepsilon^{-1} t, y\right), \quad v_{\varepsilon}(t, y)=\varepsilon^{-1} V_{\varepsilon}\left(\varepsilon^{-1} t, y\right) .
$$

The profiles $V_{\varepsilon}=V_{\varepsilon}(\theta, y)$ satisfy the singular perturbation problem

$$
\left(\partial_{\theta}+i U_{s}\right) \partial_{y} V_{\varepsilon}-i U_{s}^{\prime} V_{\varepsilon}-\varepsilon \partial_{y}^{3} V_{\varepsilon}=0
$$

on $V_{\varepsilon}(\theta, y)$. One more differentiation gives the parabolic-like equation

$$
\left(\partial_{\theta}+i U_{s}\right) \partial_{y}^{2} V_{\varepsilon}-i U_{s}^{\prime \prime} V_{\varepsilon}-\varepsilon \partial_{y}^{4} V_{\varepsilon}=0,
$$

fulfilled with the boundary conditions

$$
\left.V_{\varepsilon}\right|_{y=0}=\left.\partial_{y} V_{\varepsilon}\right|_{y=0}=\left.\partial_{y}^{3} V_{\varepsilon}\right|_{y=0}=0 .
$$

We have discretized this equation in space using finite differences on a stretched grid, and in time through a Crank-Nicholson scheme. Starting from initial random data (i.e. with random values at each grid point), we have computed its time evolution for values of $k=\varepsilon^{-1}$ ranging from 1 to $3 \times 10^{7}$. For sufficiently large times, one observes that the numerical solution $V_{\varepsilon}^{\text {num }}$ behaves like

$$
V_{\varepsilon}^{\text {num }}(\theta, y) \approx e^{i \omega^{\text {num }}(\varepsilon) \theta} v_{\varepsilon}^{\text {num }}(y)
$$

in the sense that

$$
\omega^{\text {num }}(\varepsilon):=\frac{V_{\varepsilon}^{\text {num }}(\theta+\Delta \theta, y)-V_{\varepsilon}^{\text {num }}(\theta, y)}{\Delta \theta V_{\varepsilon}^{\text {num }}(t, y)}
$$

becomes independent of $\theta$ and $y$. Computations show a relation of the type

$$
\omega^{\text {num }}(\varepsilon) \sim-U_{s}(a)+\sqrt{\varepsilon}(-0.92-0.91 i) ;
$$



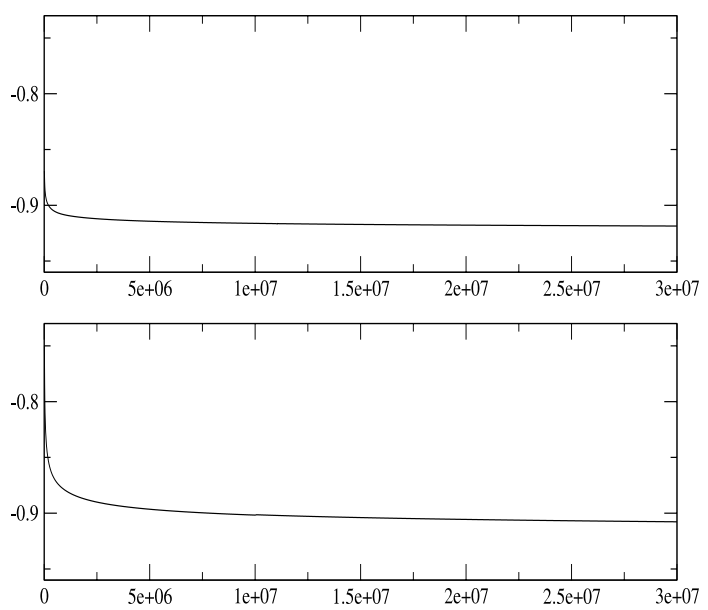

Figure 1. The correction to the inviscid eigenvalue. Plot of $\frac{1}{\sqrt{\varepsilon}}\left(\omega^{n u m}(\varepsilon)+U_{s}(a)\right)$, seen as a function of the tangential frequency $k=\varepsilon^{-1}$. Top: real part. Bottom: imaginary part. As expected from the theory, both approximately converge to -0.9 , as $k$ goes to infinity.

see Figure 1. Here $a=\frac{1}{\sqrt{2}}$ is as usual the critical point of $U_{s}$. This relation is in very good agreement with the theoretical prediction,

$$
\omega^{t h}(\varepsilon):=-U_{s}(a)+\varepsilon^{1 / 2} \frac{\left|U^{\prime \prime}(a)\right|^{1 / 2} \tau}{\sqrt{2}} \approx-U_{s}(a)+\sqrt{\varepsilon}(-0.92-0.92 i),
$$

if we take for $\tau$ the value $-0.706-0.706 i$ found in the previous subsection. Moreover, with this value of $\tau$, one can compute directly the solution $V$ of the shear layer equation. After proper rescaling, this allows for comparison between the "numerical" and "theoretical" eigenmodes. More precisely, using the notation of (2.5), one can compare the functions

$v_{\text {out }}^{\text {th }}(y):=\frac{1}{\sqrt{\varepsilon}}\left(\frac{v_{\varepsilon}^{\text {reg }}(y)}{v_{\varepsilon}^{\text {reg }}(\infty)}-\frac{v_{a}(y)}{v_{a}(\infty)}\right) \quad$ and $\quad v_{\text {out }}^{\text {num }}(y)=\frac{1}{\sqrt{\varepsilon}}\left(\frac{v_{\varepsilon}^{\text {num }}(y)}{v_{\varepsilon}^{\text {num }}(\infty)}-\frac{v_{a}(y)}{v_{a}(\infty)}\right)$

which should both describe the correction to the inviscid eigenmode outside the shear layer. As regards the shear layer, one can compare

$$
v_{i n}^{t h}(z):=\frac{1}{\sqrt{\varepsilon}} \frac{v_{\varepsilon}^{s l}\left(\varepsilon^{1 / 4}(z+a)\right)}{v_{\varepsilon}^{r e g}(\infty)}
$$

and

$$
v_{\text {in }}^{\text {num }}(z):=\frac{1}{\sqrt{\varepsilon}} \frac{v_{\varepsilon}^{\text {num }}\left(\varepsilon^{1 / 4}(z+a)\right)}{v_{\varepsilon}^{\text {num }}(+\infty)}-v_{\text {out }}^{\text {num }}\left(\varepsilon^{1 / 4}(z+a)\right)
$$


Illustrations of these comparisons are given in Figures 2 and 3. Again, we obtain an excellent agreement. This confirms that the instability mechanism we have described is indeed effective and moreover dominates the linear dynamics (1.6).
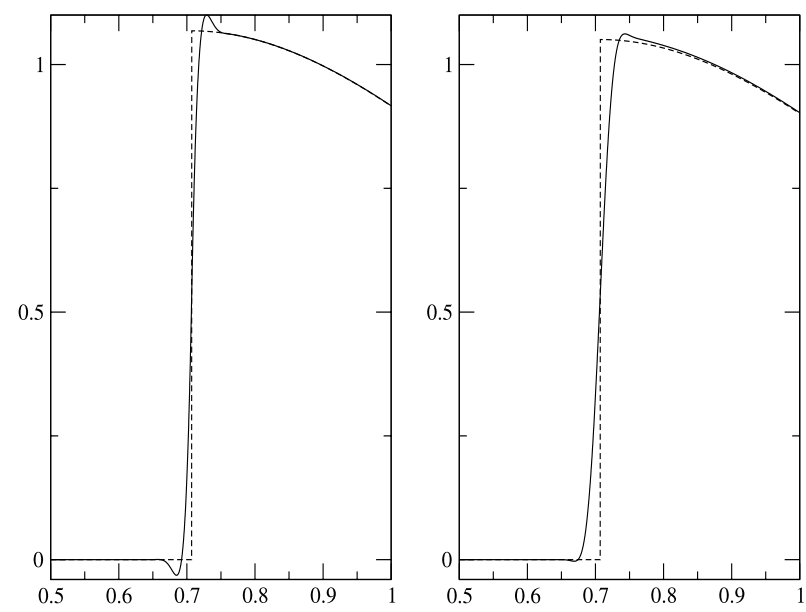

Figure 2. Plots of $v_{\text {out }}^{\text {th }}$ (dashed line) and $v_{\text {out }}^{\text {num }}$ (full line), seen as functions of $y$, at $\varepsilon=10^{-7}$. The left and right figures correspond respectively to the real and imaginary parts. They match, as expected, outside the shear layer.
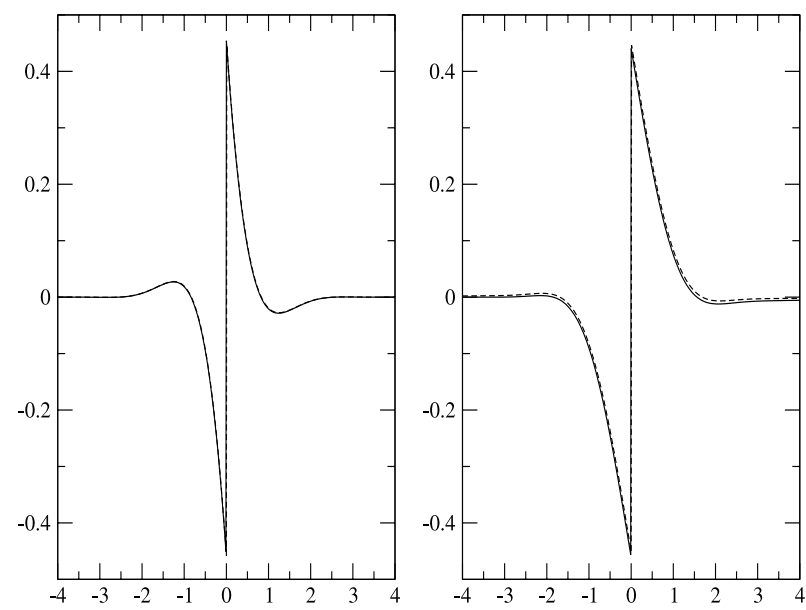

Figure 3. Plots of the shear layer corrections $v_{i n}^{t h}$ (dashed line) and $v_{i n}^{\text {num }}$ (full line), seen as functions of $z$, at $\varepsilon=10^{-7}$. The left and right figures correspond respectively to the real and imaginary parts. 


\section{Appendix: WeLl-POSEDNESS IN THE ANALYTIC SETTING}

We start from a simple estimate on the heat equation: For $U_{0} \in W_{\alpha}^{0, \infty}\left(\mathbb{R}_{+}\right)$, $F \in L^{1}\left(0, T ; W_{\alpha}^{0, \infty}\left(\mathbb{R}_{+}\right)\right)$, the solution $U$ of

$$
\partial_{t} U-\partial_{y}^{2} U=F \quad \text { on } \mathbb{R} \times \mathbb{R}_{+},\left.\quad U\right|_{t=0}=U_{0},\left.\quad U\right|_{y=0}=0,
$$

satisfies

$$
\|U\|_{L^{\infty}\left(W_{\alpha}^{0, \infty}\right)} \leq C\left(\left\|U_{0}\right\|_{W_{\alpha}^{0, \infty}}+\|F\|_{L^{1}\left(W_{\alpha}^{0, \infty}\right)}\right) .
$$

This estimate follows directly from the representation formula

$$
U(t, y)=\int_{\mathbb{R}_{+}} S(t, y, z) U_{0}(z) d z+\int_{0}^{t} \int_{\mathbb{R}_{+}} S(t-s, y, z) F(s, z) d z d s,
$$

where the heat kernel in the half-plane $S(t, y, z)$ reads

$$
S(t, y, z):=G(t, y-z)-G(t, y+z), \quad G(t, y):=\frac{1}{\sqrt{4 \pi t}} \exp \left(-y^{2} / 4 t\right) .
$$

The details are left to the reader. This estimate allows us to prove Proposition 1 . Indeed, by decomposing

$$
u(t, x, y)=\sum_{k \in \mathbb{Z}} e^{i k x} U^{k}(t, y),
$$

the well-posedness is an easy consequence of the a priori estimate

$$
\left\|U^{k}(t, \cdot)\right\|_{W_{\alpha}^{0, \infty}} \leq C e^{\rho k t}\left\|U^{k}(0, \cdot)\right\|_{W_{\alpha}^{0, \infty}}
$$

for some $\rho$. Now, the equation satisfied by $U^{k}$ is

$$
\partial_{t} U^{k}-\partial_{y}^{2} U^{k}=i k\left(U_{s}^{\prime} \int_{0}^{y} U^{k}(t, z) d z-U_{s} U^{k}\right) .
$$

Using (A.1), we get

$$
\left\|U^{k}(t, \cdot)\right\|_{W_{\alpha}^{0, \infty}} \leq C\left\|U^{k}(0, \cdot)\right\|_{W_{\alpha}^{0, \infty}}+C_{s} k \int_{0}^{t}\left\|U^{k}(s, \cdot)\right\|_{W_{\alpha}^{0, \infty}} d s,
$$

where $C_{s}$ depends on $u_{s}$. We conclude by the Gronwall lemma.

\section{ACKNOWLEDGEMENTS}

The first author would like to thank Guy Métivier, Jeffrey Rauch, and Mikael de la Salle for fruitful discussions. He acknowledges grant ANR-08-JCJC-0104-01.

\section{REFERENCES}

1. Yann Brenier, Homogeneous hydrostatic flows with convex velocity profiles, Nonlinearity $\mathbf{1 2}$ (1999), no. 3, 495-512. MR1690189 (2000b:35205)

2. Earl A. Coddington and Norman Levinson, Theory of ordinary differential equations, McGraw-Hill Book Company, Inc., New York-Toronto-London, 1955. MR0069338 (16,1022b)

3. Weinan E, Boundary layer theory and the zero-viscosity limit of the Navier-Stokes equation, Acta Math. Sin. (Engl. Ser.) 16 (2000), no. 2, 207-218. MR.1778702 (2001d:76037)

4. Weinan E and Bjorn Engquist, Blowup of solutions of the unsteady Prandtl's equation, Comm. Pure Appl. Math. 50 (1997), no. 12, 1287-1293. MR1476316 (99c:35196)

5. Emmanuel Grenier, On the derivation of homogeneous hydrostatic equations, M2AN Math. Model. Numer. Anal. 33 (1999), no. 5, 965-970. MR1726718 (2000j:76051)

6. - On the nonlinear instability of Euler and Prandtl equations, Comm. Pure Appl. Math. 53 (2000), no. 9, 1067-1091. MR.1761409 (2001i:76056) 
7. E. Guyon, J.P. Hulin, and L. Petit, Hydrodynamique physique, EDP Sciences, vol. 142, CNRS Editions, Paris, 2001.

8. Lan Hong and John K. Hunter, Singularity formation and instability in the unsteady inviscid and viscous Prandtl equations, Commun. Math. Sci. 1 (2003), no. 2, 293-316. MR1980477 (2005k:76035)

9. Tosio Kato, Perturbation theory for linear operators, Classics in Mathematics, SpringerVerlag, Berlin, 1995, Reprint of the 1980 edition. MR1335452 (96a:47025)

10. Maria Carmela Lombardo, Marco Cannone, and Marco Sammartino, Well-posedness of the boundary layer equations, SIAM J. Math. Anal. 35 (2003), no. 4, 987-1004 (electronic). MR2049030 (2005a:76137)

11. O. A. Oleinik and V. N. Samokhin, Mathematical models in boundary layer theory, Applied Mathematics and Mathematical Computation, vol. 15, Chapman \& Hall/CRC, Boca Raton, FL, 1999. MR:1697762 (2000c:76021)

12. L. Prandtl, Uber flüssigkeits-bewegung bei sehr kleiner reibung., Actes du 3ème Congrés international dse Mathématiciens, Heidelberg, Teubner, Leipzig, 1904, pp. 484-491.

13. Marco Sammartino and Russel E. Caflisch, Zero viscosity limit for analytic solutions, of the Navier-Stokes equation on a half-space. I. Existence for Euler and Prandtl equations, Comm. Math. Phys. 192 (1998), no. 2, 433-461. MR1617542 (99d:35129a)

14. Z Zero viscosity limit for analytic solutions of the Navier-Stokes equation on a halfspace. II. Construction of the Navier-Stokes solution, Comm. Math. Phys. 192 (1998), no. 2, 463-491. MR1617538 (99d:35129b)

15. Zhouping Xin and Liqun Zhang, On the global existence of solutions to the Prandtl's system, Adv. Math. 181 (2004), no. 1, 88-133. MR2020656 (2005f:35219)

DMA/CNRS, Ecole Normale Supérieure, 45 rue d'Ulm,75005 Paris, France

EnS/IPGP/CNRS, Ecole Normale Supérieure, 29 rue Lhomond, 75005 Paris, France 